\title{
VERSÃO BRASILEIRA DA ESCALA CORNELL DE DEPRESSÃO EM DEMÊNCIA (CORNELL DEPRESSION SCALE IN DEMENTIA)
}

\author{
Maria Teresa Carthery-Goulart ${ }^{1}$, Renata Areza-Fegyveres', Rodrigo R. Schultz', \\ Ivan Okamoto ${ }^{2}$, Paulo Caramelli', Paulo Henrique F. Bertolucci ${ }^{2}$, Ricardo Nitrini ${ }^{1}$
}

\begin{abstract}
RESUMO - Objetivo: Tradução e adaptação da escala Cornell de depressão em demência e verificação da confiabilidade entre e intra-examinadores da versão na língua portuguesa. Método: A versão original da Escala Cornell foi traduzida para o português por firma especializada em tradução de textos médicos e retrotraduzida para o inglês por outros dois tradutores independentes. As divergências de tradução foram identificadas e discutidas, chegando-se à versão que foi submetida à pré-teste para adaptação sócio-cultural. Após esta adaptação, obteve-se a versão final que foi administrada a amostra de 29 pacientes com doença de Alzheimer provável e aos seus cuidadores. Resultados: A versão final da escala mostrou-se de fácil aplicação e obteve boa confiabilidade intra-examinador (Kappa=0,77; $p<0,001$ ) e entre-examinadores (Kappa $=0,76 ; p<0,001)$. Conclusão: A versão brasileira da Escala Cornell é um instrumento que pode ser utilizado para avaliação e acompanhamento de depressão em pacientes com demência.
\end{abstract}

PALAVRAS-CHAVE: depressão, escalas, adaptação, reprodutibilidade dos testes.

\section{Brazilian version of the Cornell depression scale in dementia}

ABSTRACT - Objective: Translating and adapting the Cornell scale for depression in dementia to the Portuguese language and verifying the interrater and test-retest reliability of the translated and adapted version. Method: The Cornell scale was translated into Portuguese and back translated into English. Divergences of translation were identified and discussed, resulting in a version which was submitted to a pre-test for cross-cultural adaptation. The final version was administered to a sample of 29 patients with probable AD and to their caregivers. Results: The Cornell Scale presented good interrater (Kappa=0,77; $p<0,001)$ and test-retest reliability (Kappa=0,76; $p<0,001)$. The final version was easy to administer and well understood by the caregivers. Conclusion: The Brazilian version of the Cornell Scale is an instrument with good reliability to evaluate depression in patients with dementia. This tool will contribute to the evaluation and follow-up of depressed patients with dementia in our population and may also be used in multicentric studies with Brazilian population.

KEY WORDS: depression, scales, adaptation, reproducibility of results.

Depressão e demência são síndromes clínicas muito freqüentes na população idosa e podem, muitas vezes, coexistir. A prevalência de sintomas depressivos em pacientes com doença de Alzheimer (DA) varia entre 10 e $86 \%$, dependendo dos critérios diagnósticos, das avaliações utilizadas e das populações estudadas $^{1-5}$. Estudos longitudinais sugerem que a depressão pode preceder o desenvolvimento de demência ou mesmo constituir um fator de risco para o aparecimento da $D A^{6-8}$. Esses fatores apontam para a necessidade de se utilizar instrumentos específicos para avaliar sintomas depressivos em pacientes com de- mência. A escala Cornell de depressão em demência (ECDD) é um instrumento para auxiliar em pesquisa farmacológica e em estudos sobre a evolução de sintomas psiquiátricos em pacientes com demência ${ }^{9} \mathrm{e}$ mostrou-se confiável, válida e sensível de acordo com critérios de escalas psiquiátricas discutidos por Hamil$\operatorname{ton}^{10}$. Foi elaborada de forma a obter informações, não somente pelo exame clínico do paciente, mas também por meio de questionário aplicado ao cuidador. Sua utilização é indicada quando o objetivo é quantificar os sintomas e não fazer o diagnóstico, o que requer a utilização de outros instrumentos ${ }^{9}$.

\footnotetext{
${ }^{1}$ Grupo de Neurologia Cognitiva e do Comportamento (GNCC) da Clínica Neurológica do Hospital das Clínicas da Faculdade de Medicina da Universidade de São Paulo, São Paulo SP, Brasil (HCFMUSP); ${ }^{2}$ Setor de Neurologia do Comportamento do Departamento de Neurologia e Neurocirurgia da Escola de Paulista de Medicina, Universidade Federal de São Paulo, São Paulo SP, Brasil (EPM-UNIFESP).
}

Recebido 22 Setembro 2006, recebido na forma final 11 Maio 2007. Aceito 18 Junho 2007. 
Há inúmeros estudos comparativos entre escalas para depressão que incluem a ECDD ${ }^{4,11-14}$. Vida e col. ${ }^{15}$ verificaram que a ECDD pode estabelecer estádios da sintomatologia depressiva em todos os níveis de gravidade da depressão. Por esta razão, seu uso é vantajoso em relação a outras escalas, como a de Hamilton, em que há falha na distinção entre depressão leve e ausência de depressão, ou na quantificação da depressão em casos de demência grave, uma vez que os pacientes não conseguem informar os sintomas. Müller-Thomsem e col. ${ }^{4}$ concluíram que a ECDD é um dos instrumentos mais adequados para detectar depressão em pacientes com DA, independentemente da gravidade da demência. Um estudo realizado no Japão ${ }^{14}$ também chegou a conclusão semelhante. A ECDD foi utilizada em estudos com diversos objetivos, dentre os quais, o de comparar subgrupos de pacientes com demência ${ }^{16-18}$. Além disso, a ECDD tem sido utilizada em ensaios clínicos que avaliam efeito de fármacos no tratamento da depressão em demência $^{19-23}$. Em resumo, a ECDD é um instrumento de fácil e rápida aplicação, que contempla as características clínicas do paciente com demência e é capaz de quantificar os sintomas, o que é bastante útil quando são realizados estudos para verificação da eficácia de um tratamento antidepressivo nesta população.

O presente estudo visou traduzir e adaptar a ECDD e verificar a confiabilidade entre-examinadores e teste-reteste da versão adaptada e traduzida. Sua tradução e adaptação transcultural para a Língua Portuguesa viabilizará seu uso em nosso meio.

\section{MÉTODO}

A versão original da $\mathrm{ECDD}^{13}$ foi traduzida para a língua portuguesa por uma empresa especializada em tradução de textos médicos. Esta versão foi, em seguida, traduzida de volta para a língua inglesa, de modo independente, por dois neurologistas experientes em escalas para demência e fluentes em língua inglesa. As diferenças de tradução foram identificadas e discutidas em painel, considerando as razões para as diferenças e cotejando-as com a forma original em língua inglesa, chegando-se a uma versão que foi submetida a um pré-teste para a adaptação sócio-cultural. Nesta fase, oito indivíduos atendidos pelo Grupo de Neurologia Cognitiva e do Comportamento da Faculdade de Medicina da USP (GNCC HCFMUSP), que preencheram os critérios do NINCDS-ADRDA ${ }^{24}$ para DA provável de graus leve e moderado, foram avaliados e seus cuidadores, entrevistados. Durante a aplicação dos questionários, solicitouse que cada cuidador explicasse seu entendimento sobre cada pergunta. A partir deste levantamento, verificamos nenhuma questão suscitou dúvidas a cuidadores de diferentes níveis de escolaridade, portanto não era necessário introduzir alterações maiores de linguagem. As escalas foram aplicadas por três neurologistas e uma fonoaudiólo- ga, todos com experiência na aplicação de escalas para indivíduos com demência. A versão final da escala, incluindo as normas de aplicação está disponível em www.abneuro.org/departamento cientifico/Neurologia Cognitiva e do Envelhecimento.

Posteriormente, esta versão foi administrada a uma amostra de 29 pacientes com DA provável segundo os critérios do NINCDS-ADRDA ${ }^{24}$, com pontuações no Mini-Exame do Estado Mental ${ }^{25,26}$ entre 14 e 26 pontos (correspondendo à demência leve a moderada), atendidos nos ambulatórios do GNCC-HCFMUSP e do Setor de Neurologia do Comportamento da Universidade Federal de São Paulo (UNIFESP). Vinte e quatro pacientes fizeram parte da amostra para checar a confiabilidade entre-examinadores e 20 pacientes, para checar a confiabilidade teste-reteste, com intervalo de uma a duas semanas entre a primeira e a segunda avaliação.

Ética - O presente estudo teve aprovação da Comissão de Ética para Análise de Projetos de Pesquisa da Diretoria Clínica do Hospital das Clínicas e da Faculdade de Medicina da Universidade de São Paulo e do Comitê de Ética em Pesquisa da Universidade Federal de São Paulo e todos os pacientes e/ou seus responsáveis legais assinaram Termo de Consentimento informado para a aplicação das escalas.

Análise estatística - A confiabilidade inter-examinador foi testada com a estatística de Kappa, utilizando o software Statistical Package for the Social Sciences (SPSS 10.0), assim como a confiabilidade entre-examinadores, a fim de comparar as pontuações dos dois examinadores. O teste de Spearman foi utilizado para determinar a correlação.

\section{RESULTADOS}

A Tabela apresenta as características demográficas dos pacientes avaliados.

A versão final da escala foi de fácil aplicação e entendimento, levando aproximadamente 30 minutos para ser administrada.

A ECDD teve boa confiabilidade teste-reteste (Kappa 0,76; $p<0,001$ ) e entre-examinadores (Kappa $0,76 ; p<0,001)$. A correlação de Spearmann foi 0,87 $(p<0,001)$ para entre-examinadores e 0,85 $(p<0,001)$ para intra-examinadores. A pontuação média obtida na primeira avaliação foi 5,42 e o desvio-padrão 5,23 .

Tabela. Características demográficas dos pacientes avaliados

\begin{tabular}{lc}
\hline Características & Pacientes (29) \\
\hline Sexo & $14 \mathrm{M} 15 \mathrm{~F}$ \\
Idade - média (DP) & $74,1(8,7)$ \\
Escolaridade - média (DP) & $6,8(4,8)$ \\
MEEM - média (DP) & $19,9(3,8)$ \\
\hline
\end{tabular}

$M$, masculino; F, feminino; DP, desvio-padrão; MEEM, Mini-Exame do Estado Mental. 


\section{DISCUSSÃO}

A ocorrência de síndrome demencial potencialmente reversível durante um episódio depressivo, a instalação do primeiro episódio depressivo na senescência e achados anormais de neuroimagem, como atrofia cortical e alargamento de ventrículos, são fatores preditivos importantes para o aparecimento de demência ${ }^{27,28}$. Em idosos, o tempo do episódio depressivo correlaciona-se com a redução do volume hipocampal, atrofia de substância cinzenta do córtex pré-frontal e perda de células gliais ${ }^{29,30}$. Dessa forma, torna-se importante haver no nosso meio escalas que identifiquem e graduem sintomas depressivos.

A maioria das escalas de depressão existentes baseia-se em informações fornecidas pelos pacientes que, ou completam um questionário, ou são entrevistados por um profissional de saúde devidamente treinado. Este método torna difícil a avaliação de pacientes com demência, já que suas falhas de concentração, memória e julgamento podem prejudicar a interpretação de suas respostas.

A ECDD mostrou-se de fácil e rápida aplicação, levando em média vinte minutos para a avaliação do cuidador/familiar e dez minutos para avaliação do paciente. Esses dados, quando comparados ao estudo original ${ }^{9}$, mostram-se semelhantes. A versão traduzida e adaptada foi avaliada em suas propriedades de medida, mostrando-se uma escala estável e confiável, em relação à reprodutibilidade tanto pelo mesmo examinador em dois momentos distintos, quanto entre diferentes examinadores.

Nas últimas décadas tem ocorrido tendência crescente ao desenvolvimento de pesquisas multicêntricas. Estabeleceu-se, então, a necessidade de serem elaboradas medidas específicas para utilização das diversas escalas em outros idiomas, além do de sua origem, tanto para o emprego dos mesmos em outros países, quanto em populações de imigrantes, uma vez que em ambas as situações podem estar presentes diferenças culturais importantes ${ }^{31,32}$. A realização de estudos multicêntricos nos possibilita avaliar um mesmo fenômeno em diversos meios, identificando diferenças sócio-culturais entre populações afetadas por uma mesma doença. Para isso devemos considerar que o instrumento de avaliação utilizado seja no mínimo similar, de forma a permitir que os resultados possam ser comparados.

Em diferentes meios culturais, a adaptação transcultural é um pré-requisito para podermos pesquisar um mesmo fenômeno ${ }^{31}$. A adaptação transcultural de um instrumento de avaliação pressupõe uma metodologia que engloba etapas que vão além da simples tradução do instrumento, considerando aspectos culturais e de linguagem da população-alvo. Neste estudo, a ECDD foi traduzida e adaptada para população em questão de acordo com a metodologia proposta por Guillemin e col. ${ }^{31}$.

O estudo de validade da versão original foi baseado em comparações entre os escores na ECDD e os diagnósticos dados por psiquiatras por meio dos "Critérios Diagnósticos de Pesquisa" (Research Diagnostic Criteria-RDC). Estes últimos se baseiam na história do paciente obtida no prontuário médico, na consulta com o psiquiatra do paciente, quando possível, e do exame clínico, consistindo num sistema categórico de pontuação e não em uma escala quantitativa como a ECDD ${ }^{9,10}$. Acreditamos que a versão brasileira da ECDD possa ser útil para a realização de estudos em nossa população e também para permitir a participação de grupos brasileiros de pesquisa clínica em projetos cooperativos multicêntricos internacionais que empreguem esta escala.

\section{REFERÊNCIAS}

1. Burns A. Neuropsychiatric phenomena in Alzheimer's disease. J Neurol Neurosurg Psychiatry 2001;71:715.

2. Almeida O P. Sintomas psiquiátricos entre pacientes com demência atendidos em um serviço ambulatorial. Arq Neuropsiquiatr 1999;57:937-943.

3. Zubenko GS, Zubenko WN, McPherson S, et al. A collaborative study of the emergence and clinical features of major depressive syndrome of Alzheimer's disease. Am J Psychiatry 2003;160:857-866.

4. Muller-Thomsen T, Arlt S, Mann U, Mass R, Ganzer S. Detecting depression in Alzheimer's disease: evaluation of four different scales. Arch Clin Neuropsychol 2005;20:271-276.

5. Tatsch MF, Bottino CM, Azevedo D, et al. Neuropsychiatric symptoms in Alzheimer's disease and cognitively impaired, nondemented elderly from a community-based sample in Brazil: prevalence and relationship with dementia severity. Am J Geriatr Psychiatry 2006;14:438-445.

6. Alexopoulos GS, Buckwalter K, Olin J, Martinez R, Wainscott C, Krishnan KR. Comorbidity of late life depression: an opportunity for research on mechanisms and treatment. Biol Psychiatry 2002;52:543-558.

7. Ownby RL, Crocco E, Acevedo A, John V, Loewenstein D. Depression and risk for Alzheimer disease: systematic review, meta-analysis, and metaregression analysis. Arch Gen Psychiatry 2006;63:530-538.

8. Yochim BP, MacNeill SE, Lichtenberg PA. "Vascular depression" predicts verbal fluency in older adults. J Clin Exp Neuropsychol 2006;28:495-508.

9. Alexopoulos GS, Abrams RC, Young RC, Shamoian CA. Cornell Scale for Depression in dementia. Biol Psychiatry 1988;23:271-284.

10. Hamilton M. General problems of psychiatric rating scales (especially for depression). Mod Probl Pharmacopsychiatry 1974;7:125-138.

11. Camus V, Schmitt L, Ousset PJ, Micas M. Dépression et démence: contribution à la validation française de deux échelles de depression: "Cornell Scale for Depression in Dementia" et "Dementia Mood assessment Scale". L'Encephale 1995;21:201-208.

12. Bonin-Guillaume S, Clément JP, Chassain AP, Léger JM. Évaluation psychométrique de la depression du sujet âgé: quels instruments? Quelles perspectives d'avenir? L'Encéphale 1995;21:25-34.

13. Schreiner AS, Hayakawa H, Morinoto T, Kakuma T. Screening for late life depression: cut-off scores for the Geriatric Depression Scale and the Cornell Scale for Depression in Dementia among Japanese subjects. Int J Geriatr Psychiatry 2003;18:498-505.

14. Lam CK, Lim PPJ, Low BL, Chiam PC, Sahadevan S. Depression in dementia: a comparative and validation study of four brief scales in the elderly Chinese. Int J Psychiatry 2004;19:422-428.

15. Vida S, Des Rosiers P, Carrier L, Gauthier S. Depression in Alzheimer's disease: receiver operating characteristic analysis of the Cornell Scale for Depression in Dementia and the Hamilton Depression Scale. J Geriatr Psychiatry Neurol 1994;7:159-162. 
16. Ballard CG, O'Brien JT, Swann AG, Thompson P, Neill D, McKeith IG. The natural history of psychosis and depression in dementia with Lewy bodies and Alzheimer's disease: persistence and new cases over 1 year of follow-up. J Clin Psychiatry 2001;62:46-49.

17. Nambudiri DE, Teusink JP, Fensterheim L, Young RC. Age and psychosis in degenerative dementia. Int J Geriatr Psychiatry 1997;12:11-14.

18. Simpson S, Allen H, Tomenson B, Burns A. Neurological correlates of depressive symptoms in Alzheimer's disease and vascular dementia. J Affec Dis 1999;53:129-136.

19. Katona CL, Hunter BN, Bray J. A double-blind comparison of the efficacy and safety of paroxetine and imipramine in the treatment of depression with dementia. Int J Geriatr Psychiatry 1998;13:100-108.

20. Lyketsos CG, Sheppard JM, Steele CD, et al. Randomized, placebo-controlled, double-blind clinical trial of sertraline in the treatment of depression complicating Alzheimer's disease: initial results from the depression in Alzheimer's disease study. Am J Psychiatry 2000;157:1686-1689.

21. Brodaty H, Draper BM, Millar J, et al. Randomized controlled trial of different models of care for nursing home residents with dementia complicated by depression or psychosis. J Clin Psychiatry 2003;64:63-72.

22. Roose SP, Nelson JC, Salzman C, Hollander SB, Rodrigues H. Mirtazapine in the Nursing Home Study Group. Open-label study of mirtazapine orally disintegrating tablets in depressed patients in the nursing home. Curr Med Res Opin 2003;19:737-746.

23. Lyketsos CG, DelCampo L, Steinberg M, et al. Treating depression in Alzheimer's disease: efficacy and safety of sertraline therapy, and the benefits of depression reduction: the DIADS. Arch Gen Psychiatry 2003;60:737-746.

24. McKhann G, Drachman D, Folstein M, Katzman R, Price D, Stadlan EM.
Clinical diagnosis of Alzheimer's disease: report from the NINCDSADRDA Work Group under the auspices of Department of Health and Human Services Task Force on Alzheimer's Disease. Neurology 1984;34:939-944.

25. Folstein M, Folstein S, McHugh PR. Mini-mental state: a practical method for grading the cognitive state of patients for the clinician. J Psychiatr Res 1975;12:198-198.

26. Brucki SM, Nitrini R, Caramelli P, Bertolucci PH, Okamoto IH. Suggestions for utilization of the mini-mental state examination in Brazil. Arq Neuropsiquiatr 2003;61:777-781.

27. Alexopoulos GS, Chester JG. Outcomes of geriatric depression. Clin Geriatr Med 1992;8:363-376.

28. Steffens DC, Otey E, Alexopoulos GS, Butters MA, et al. Perspectives on depression, mild cognitive impairment, and cognitive decline. Arch Gen Psychiatry 2006;63:130.

29. Bell-McGinty S, Butters MA, Meltzer CC, Greer PJ, Reynolds CF, Becker JT. Brain morphometric abnormalities in geriatric depression: long-term neurobiological effects of illness duration. Am J Psychiatry 2002;159:1424-1427.

30. MacQueen GM, Campbell S, McEwen BS et al. Course of illness, hippocampal function, and hippocampal volume in major depression. Proc Natl Acad Sci USA 2003;100:1387-1392.

31. Guillemin F, Bombardier C, Beaton D. Cross-cultural adaptation of health-related quality of life measures: literature review and proposed guidelines. J Clin Epidemiol 1993;46:1417-1432.

32. Duarte YAO. Família: rede de suporte ou fator estressor. A ótica de idosos e cuidadores familiares. Tese. Universidade de São Paulo, 2001. 